\title{
Changes in fatty acid lengths of ceramides toward shorter chain dominance in human psoriasis skin
}

Bo-Kyung Kim, M.D.\#, Dong-In Keum, M.D. ${ }^{*}$, Jong Cheol Shon++, Kwang-Hyeon Liu, Ph.D.++, Seung-Phil Hong, M.D., Ph.D.+, Sung Ku Ahn, M.D., Ph.D.\#

\#Department of Dermatology, Yonsei University Wonju College of Medicine, Wonju; +Department of Dermatology, College of Medicine, Dankook University, Cheonan; ++College of Pharmacy and Research Institute of Pharmaceutical Sciences, Kyungpook National University, Daegu; *Central Physical Examination Center of Military Manpower Administration, Daegu, Republic of Korea

\section{Introduction}

\section{Background:}

Ceramides(CERs) are major component of the intercellular lipids, and average fatty $\operatorname{acid}(\mathrm{FA})$ chain length of the CERs correlate with the barrier function. Changes in skin lipid profiles have been associated with abnormal barrier function permeability in psoriasis. However, it is unknown how lipid synthetic enzymes affect the synthesis of ceramides.

Objective:

We aimed to identify the alteration of FA chain length of CERs and lipid synthetic enzymes including elongases(ELOVLs) in human psoriasis skin.

\section{Materials and Methods}

\section{Subjects}

$>$ Human

- Psoriasis group(n=12, 9 men and 3 women; aged 22-91 years)

- Control group: Age- and sex-matched healthy individuals without skin disorders

$>$ Animal: BALB/c female mice (5 weeks of age)

- IMQ-induced psoriasis mimicking model: apply $62.5 \mathrm{mg}$ of imiquimod $5 \%$ cream (Aldara ${ }^{@}, 3 \mathrm{M}$ pharmaceuticals) on their shaved back skin and right ear once a day for 5 days $(n=10)$

- Control group: treated with petrolatum in the same manner $(n=5)$

$>$ IRB approval: This study was approved by the ethical committee of Yonsei Wonju University of Medicine

Evaluations

- Stratum corneum(SC) sampling from lesional and non-lesional skin of patients or on the inner forearms of controls using film tape

- Skin tissue sampling from lesional skin of human and back of mice

- The unbound CERs in SC were quantified by matrix-assisted laser desorption/ionization time-of-flight mass spectrometry.

- The epidermal mRNA levels of following enzymes and transcription factors were measured by real-time quantitative PCR analysis

- The epidermal protein levels of ELOVL and Ceramide synthase(CerS) were identified by Immunohistochemistry-staining.

Statistical analysis: All data were expressed as mean \pm SD. For comparisons, Student's t-test was used. Statistical significance is a p-value of less than 0.05 .

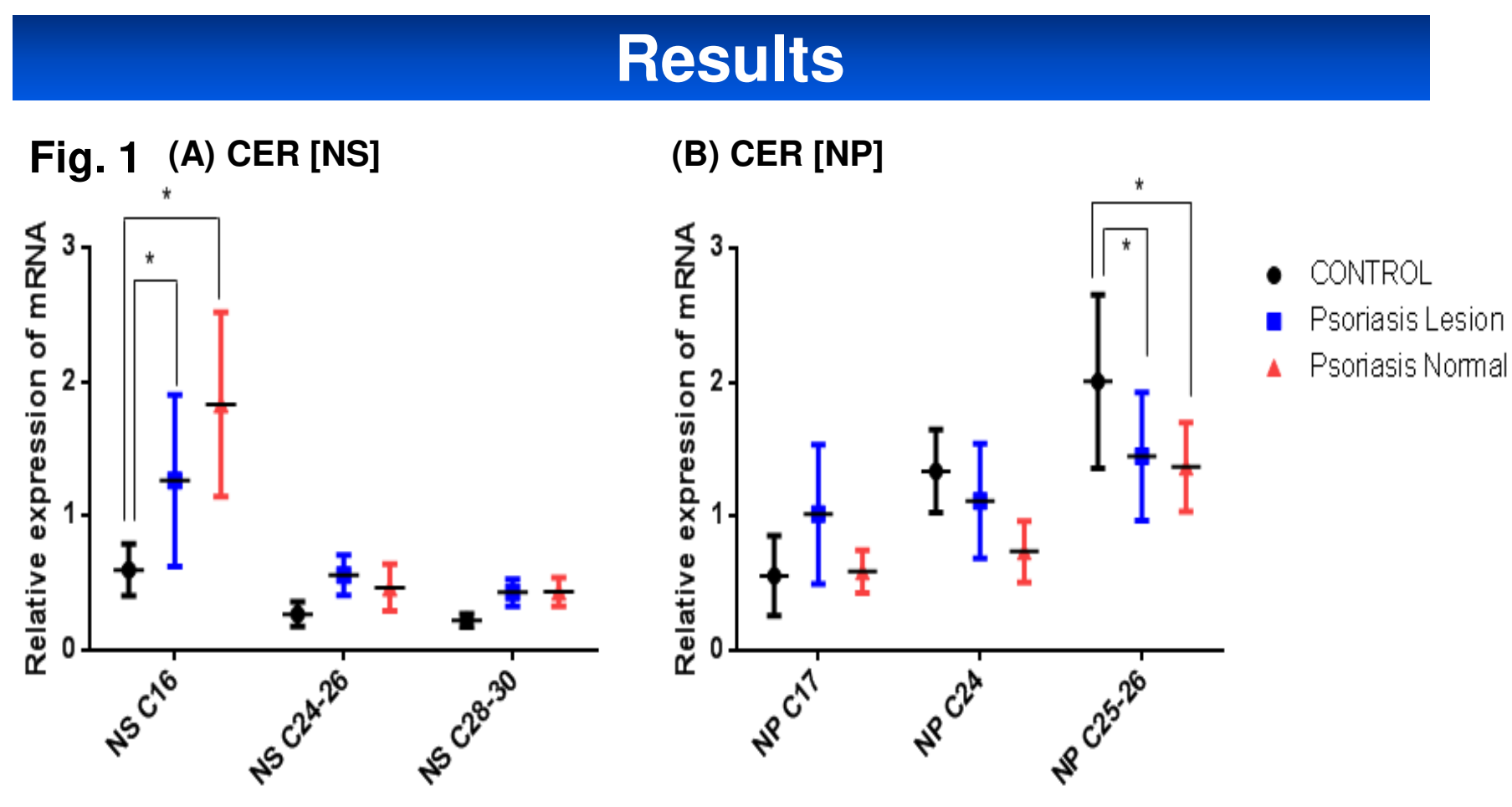

Fig 1. Amount of CER species in human epidermis

(A) CER[NS] with FAs shorter than C24 showed significantly increased level in lesional and non-lesional skin of psoriasis group

(B) CER[NP] with very long FAs (longer than C25) showed significantly

decreased level in lesional and non-lesional skin of psoriasis group $\left({ }^{*}: \mathrm{P}<0.05\right)$

Fig. 2

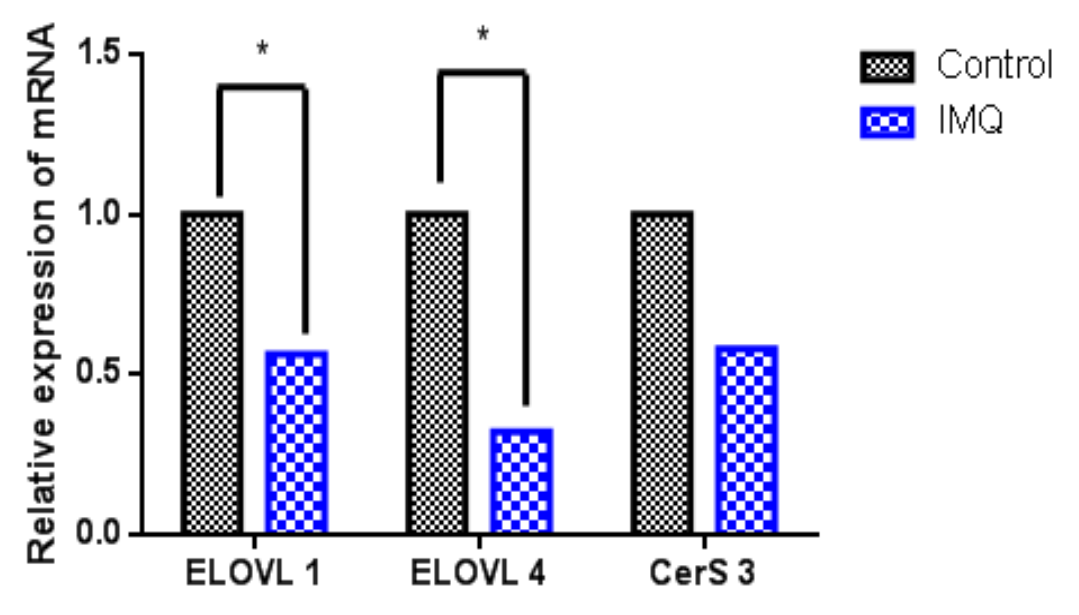

Fig 2. The mRNA levels of ELOVL1, ELOVL4 and CerS3 in mice epidermis. The mRNA level of ELOVL1, ELOVL4 and CerS3 showed significantly decreased in IMQ-treated group $\left.{ }^{*}: \mathrm{P}<0.05\right)$

\section{Fig. 3 (A) ELOVL1}

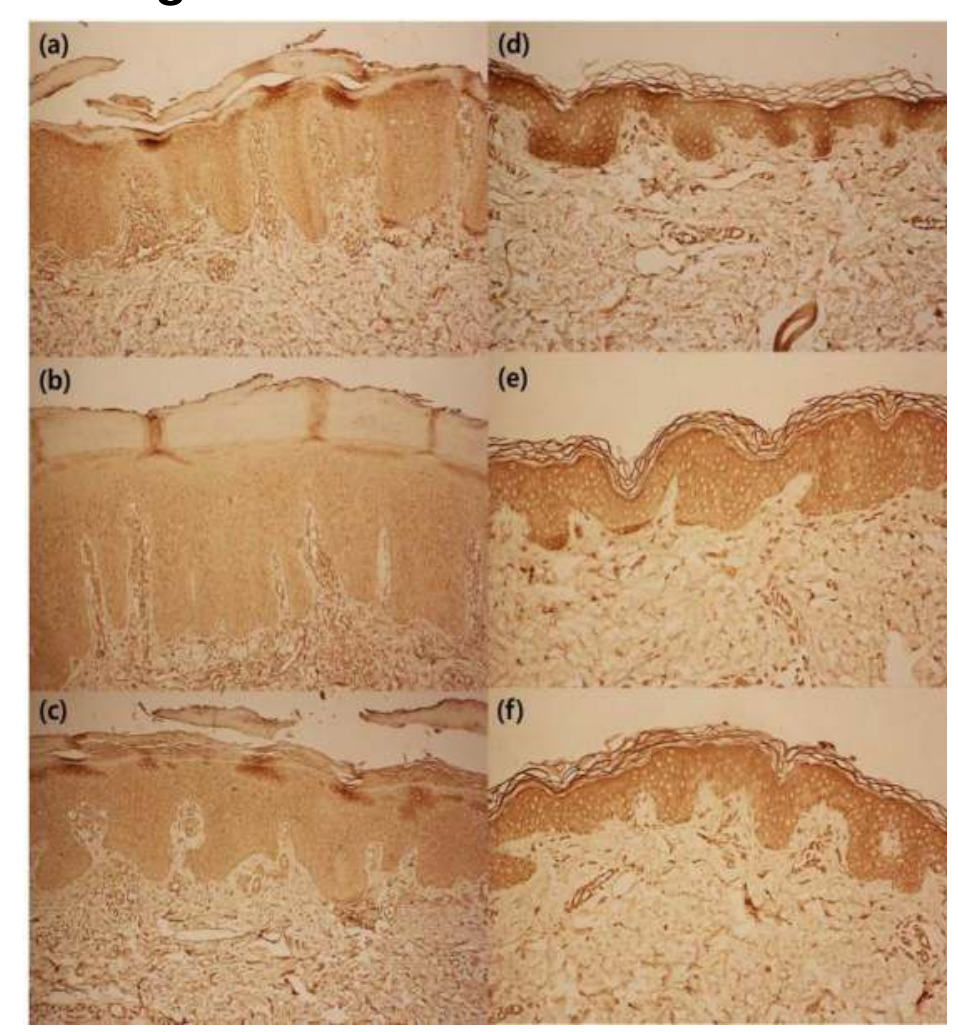

(B) CerS3

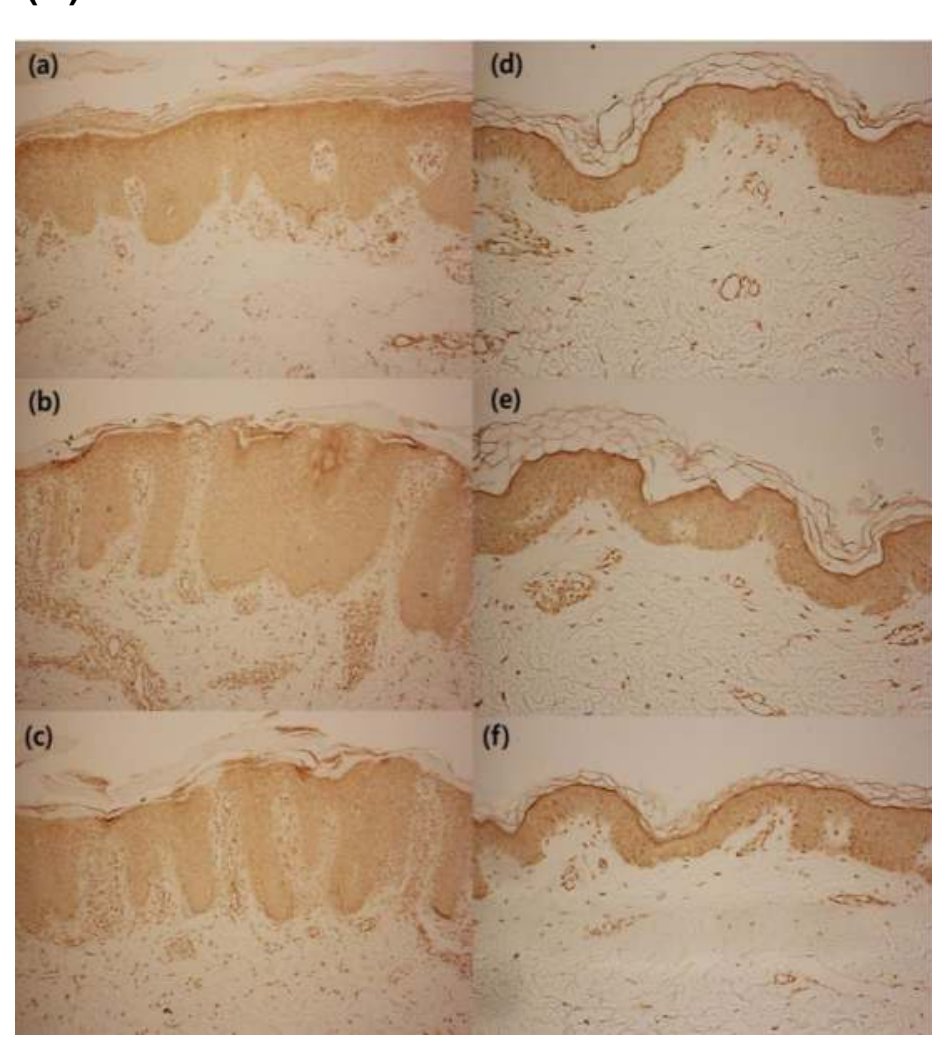

Fig 3. The immunohistochemical staining for ELOVL1 and CerS3 in human epidermis.

(A) IHC staining showed decreased level of ELOVL 1 in lesional epidermis from psoriasis(a,b,c) than control(d,e,f)

(B) IHC staining showed decreased level of CerS 3 in lesional epidermis from psoriasis $(\mathrm{a}, \mathrm{b}, \mathrm{c})$ than control $(\mathrm{d}, \mathrm{e}, \mathrm{f})$

\section{Discussion and Conclusions}

- Profiles of FA lengths of CERs shifted toward shorter chain in lesional and non-lesional skin from psoriasis

$\checkmark$ The unbound CERs ([NS],[NP]) with very long FAs (longer than C24) decreased whereas those with shoter FAs(shorter than C24) increased

in psoriasis human skin.

- The protein level of ELOVL1 and CerS3 were decreased in the IHC staining from psoriasis human skin

$\checkmark$ The mRNA expression of ELOVL1, 4 and CerS3 were also decreased

in psoriasis-like skin lesion in mice.

- The down-regulation of ELOVL 1 and CerS3 might cause impairment in the synthesis of very long chain CERs.

$\checkmark$ ELOVLS and CerSs play critical roles in regulating the length and degree of FAs.

$\checkmark$ ELOVL1 are required for generation of long-chain fatty acids(FAs) longer than C22.

$\checkmark$ CerS3 expression is associated with the appearance of the very longchain fatty acids(FAs)

Thus, we suggest that the profile changes of FA lengths of CERs mediated by enzymatic changes, especially ELOVLs, can be associated in part with barrier dysfunction in psoriasis.

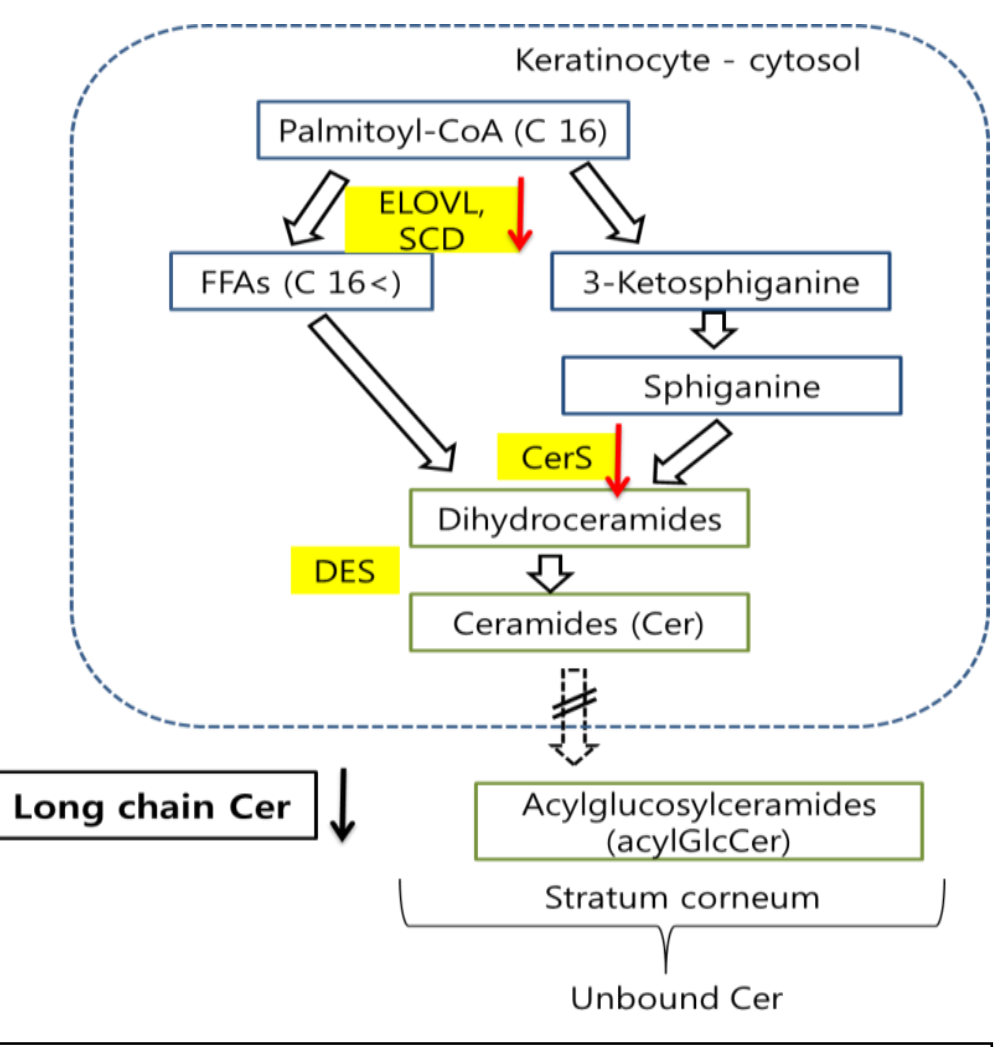

Fig 4. Schematic summary of the results

\section{Acknowledgement}

This study was supported by a grant of the Korea Healthcare technology R\&D Project Ministry of Health \& Welfare, Republic of Korea (HN11C0048).

\section{References}

Nakajima $\mathrm{K}$, Terao $\mathrm{M}$, Takaishi $\mathrm{M}$ et al. Barrier abnormality due to ceramide deficiency leads to psoriasiform inflammation in a mouse model. $J$ Invest Dermatol 2013; 133: 2555-65.

Coderch L, Lopez O, de la Maza A et al. Ceramides and skin function. Am J Clin Dermatol 2003; 4: 107-29

Tawada C, Kanoh H, Nakamura $\mathrm{M}$ et al. Interferon-gamma decreases ceramides with longchain fatty acids: possible involvement in atopic dermatitis and psoriasis. $J$ Invest Dermatol 2014; 134: 712-8.

Wolf $\mathrm{R}$, Orion $\mathrm{E}$, Ruocco $\mathrm{E}$ et al. Abnormal epidermal barrier in the pathogenesis of psoriasis. Clin Dermatol 2012; 30: 323-8. 